\title{
Multimodal treatment for local recurrent malignant gliomas: Resurgery and/or reirradiation followed by chemotherapy
}

\author{
ARSELA PRELAJ $^{1 *}$, SARA ELENA REBUZZI $^{2 *}$, MASSIMILIANO GRASSI $^{2}$, JULIO RODRIGO GIRÒN BERRİOS ${ }^{1}$, \\ SILVIA PECORARI ${ }^{1}$, CARMELA FUSTO ${ }^{3}$, CARLA FERRARA $^{4}$, MAURIZIO SALVATI $^{5}$, \\ VALERIA STATI $^{6}$, SILVERIO TOMAO $^{1,7}$ and VINCENZO BIANCO ${ }^{1}$ \\ ${ }^{1}$ Department of Medical Oncology Unit A, Policlinico Umberto I, 'Sapienza' University of Rome, I-00161 Rome; \\ ${ }^{2}$ Department of Medical Oncology, Ospedale Policlinico San Martino IST, I-16132 Genoa; ${ }^{3}$ Department of Radiological, \\ Oncological and Anatomo-Pathological Sciences, 'Sapienza' University of Rome, Policlinico Umberto I, I-00161 Rome; \\ ${ }^{4}$ Department of Public Health and Infectious Diseases, 'Sapienza' University of Rome, I-00185 Rome; ${ }^{5}$ Department of \\ Neurosurgery, IRCCS Neuromed, I-86077 Pozzilli; ${ }^{6}$ Department of Medico-Surgical Sciences and Biotechnologies, \\ 'Sapienza' University of Rome, I-00185 Rome; ${ }^{7}$ Department of Radiological Sciences, \\ Oncology and Pathology, 'Sapienza' University of Rome, I-04100 Latina, Italy
}

Received March 7, 2018; Accepted July 5, 2018

DOI: $10.3892 / \mathrm{mco} .2018 .1745$

\begin{abstract}
The therapeutic management of recurrent malignant gliomas (MGs) is not determined. Therefore, the efficacy of a multimodal approach and a combination systemic therapy was investigated. A retrospective analysis of $26 \mathrm{MGs}$ patients at first relapse treated with multimodal therapy (chemotherapy plus surgery and/or reirradiation) or chemotherapy alone was performed. Second-line chemotherapy consisted of fotemustine (FTM) in combination with bevacizumab (BEV) (cFTM/BEV) or followed by third-line BEV (sFTM/BEV). Subgroup analyses were performed. Multimodal therapy provided a higher overall response rate (ORR) (73 vs. 47\%), disease control rate (DCR) (82 vs. 67\%), median progressionfree survival (mPFS) (11 vs. 7 months; $\mathrm{P}=0.08)$ and median overall survival (mOS) (13 vs. 8 months; $\mathrm{P}=0.04$ ) compared with chemotherapy. Concomitant FTM/BEV resulted in higher ORR (84 vs. 36\%), DCR (92 vs. 57\%), mPFS (10 vs. 5 months; $\mathrm{P}=0.22)$ and $\operatorname{mOS}(11$ vs. 5.2 months; $\mathrm{P}=0.15$ ) compared with sFTM/BEV. Methylated patients did not experience additional survival benefits with multimodality treatment but had higher mPFS (10 vs 7.1 months; $\mathrm{P}=0.33$ ) and $\operatorname{mOS}$ (11 vs. 8 months; $\mathrm{P}=0.33$ ) with $\mathrm{cFTM} / \mathrm{BEV}$. Unmethylated patients experienced the greatest survival benefit with the multimodal approach
\end{abstract}

Correspondence to: Dr Arsela Prelaj, Department of Medical Oncology Unit A, Policlinico Umberto I, 'Sapienza' University of Rome, Viale del Policlinico 155, I-00161 Rome, Italy

E-mail: arsela20@hotmail.it

*Contributed equally

Key words: recurrent malignant gliomas, recurrent glioblastomas, multimodal, surgery, radiotherapy, chemotherapy, fotemustine, bevacizumab
(mPFS: 10 vs. 5 months; mOS 11 vs 6 months; both $\mathrm{P}=0.02$ ) and cFTM/BEV (mPFS: 5 vs. 2 months; mOS 6 vs. 3.2 months; both $\mathrm{P}=0.01$ ). In conclusion, in recurrent MGs, multimodal therapy and cFTM/BEV provide survival and response benefits. Methylated patients benefit from a cFTM/BEV but not from a multimodal approach. Notably, unmethylated patients had the highest survival benefit with the two strategies.

\section{Introduction}

Malignant gliomas (MGs) are the most common primary malignant brain tumours and include anaplastic gliomas (AG) and glioblastoma multiforme (GBM) (1). Maximal safe surgical resection followed by radiotherapy with concomitant and adjuvant temozolomide (TMZ) is the standard first-line treatment of GBM (2), leading to a median overall survival (mOS) of 12-15 months (3). Despite the optimal standard treatment, the local infield recurrence rate remains high ( $90 \%)$, and despite the molecular advances, no standard therapies are established for recurrent MGs. Different options are under investigation, including resurgery, reirradiation and chemotherapy, as well as their combinations $(3,4)$.

A recent review of the literature (5) showed a survival benefit and an improved functional status after resurgery followed by adjuvant treatments, with a higher OS in selected patients with favourable clinical and radiological characteristics at the time of recurrence. Preoperative Karnofsky Performance Status (KPS $>70 \%)$ and age ( $<60$ years) are important predictors of longer survival (5-7). Multiple studies have also demonstrated that a greater extent of resection is associated with better survival outcomes (8-10). However, prospective data are lacking to confirm resurgery as an independent predictor of survival $(11,12)$.

Focal radiotherapy is a similarly controversial option due to the lack of prospective randomised trials and the risk of toxicity, regarding radionecrosis and neurocognitive impairment. Recent 
advances in radiotherapy techniques, including stereotactic and hypofractionated treatments, allow for more precise treatment, sparing healthy surrounding tissue and reducing late toxicity (13). Younger age ( $<70$ years) and good performance status (PS) (KPS $>60 \%$ ) are the most important predictors of longer survival for reirradiation (14). Multiple trials have studied the combination of radiotherapy and systemic therapy, such as bevacizumab $(\mathrm{BEV})$ and TMZ $(13,15,16)$. Proton-beam therapy (PBT), a type of radiation treatment, has the advantage over photon-therapy of sparing considerable volumes of previously irradiated healthy tissue $(13,15)$. Survival and clinical benefits of PBT, alone or in association with chemotherapy, have been studied in newly diagnosed and recurrent MGs (17-20).

Many clinical trials on recurrent GBM studied the efficacy of single and/or combined chemotherapy agents, including nitrosoureas, and of targeted therapies, such as BEV, alone or associated with chemotherapy, with encouraging results $(6,21,22)$. Nitrosoureas, mainly fotemustine (FTM) (23), have been employed either in monotherapy or in combination with other agents (21), including BEV, showing potential survival benefit $(21,24-27)$.

Improved outcomes with a multimodality management of recurrent MGs have been reported in a few trials (28-30), but no standard treatment algorithm has been defined.

The aim of this study is to analyse the efficacy of the multimodal treatment as a combination of chemotherapy, as FTM and BEV in combination or in sequence, and resurgery and/or reirradiation, including PBT, in MGs patients at first recurrence.

\section{Patients and methods}

Study population. This study was conducted at the Department of Medical Oncology of Policlinico Umberto I of Rome and Latina, both of Sapienza University of Rome. The study was approved by the Institutional Review Board of Latina.

From August 2011 to August 2017, we retrospectively analysed recurrent MGs patients at first relapse treated with multimodal therapy as a combination of resurgery and/or reirradiation, including PBT, followed by chemotherapy or chemotherapy alone. All patients underwent first-line therapy with surgery followed by radio-chemotherapy according to Stupp protocol (2).

The initial diagnosis was established by magnetic resonance imaging (MRI) and histologically using WHO criteria (31). Diagnosis of recurrence was assessed by MRI in all patients and by histological examination when resurgery was performed. Clinical data included patients' characteristics, tumour characteristics and treatment information (Table I).

Treatment plan. At first recurrence, patients received either a multimodal therapy consisting of chemotherapy preceded by resurgery and/or reirradiation or chemotherapy alone. Resurgery consisted of maximal safe surgical resection. Reirradiation, including radiotherapy or PBT, was given prior to chemotherapy and after surgery. Reirradiation consisted of fractionated stereotactic radiotherapy (total dose of $60 \mathrm{~Gy}$ in 1.8 to 2.0 Gy fractions).

Chemotherapy consisted of FTM as second-line therapy in combination with BEV (concomitant FTM/BEV; cFTM/BEV) or as second-line therapy followed by third-line BEV (sequential FTM/BEV; sFTM/BEV).

The sequential treatment FTM, according to the Addeo schedule (23), consisted of an induction phase dose of $80 \mathrm{mg} / \mathrm{mq}$ every 2 weeks for 5 consecutive administrations followed by a 4-week rest period and a maintenance phase dose of $80 \mathrm{mg} / \mathrm{mq}$ every 4 weeks. BEV was administered at $10 \mathrm{mg} / \mathrm{kg}$ every 2 weeks, in off-label use. In patients who underwent resurgery, BEV commenced 4-6 weeks after surgery.

The cFTM/BEV therapy, according to the Soffietti schedule (24), consisted of an induction phase with BEV at $10 \mathrm{mg} / \mathrm{kg}$ on days 1 and 15 and FTM at $75 \mathrm{mg} / \mathrm{mq}$ on days 1 and 8 , followed by a 3 -week rest period and a maintenance phase with BEV at $10 \mathrm{mg} / \mathrm{kg}$ and FTM at $75 \mathrm{mg} / \mathrm{mq}$ every 3 weeks.

Response evaluation. Radiological evaluations consisted of 3-Tesla MRI scans (contrast-enhanced T1-weighted, T2/FLAIR-weighted, perfusion-weighted and diffusionweighted scans and MR spectroscopy). MRI evaluations were made at baseline, between each treatment modality, after the first 2 cycles of BEV or after the induction phase of FTM and then after every two cycles of BEV or FTM in the maintenance phase. Evaluation response was assessed according to RANO criteria (32) as complete (CR) and partial (PR) response, stable (SD) and progression (PD) disease. Overall response rate (ORR) was defined as the sum of $\mathrm{CR}$ and $\mathrm{PR}$ and disease control rate (DCR) was defined as the sum of CR, PR and SD.

Statistical analysis. Survival analysis was conducted on the efficacy of multimodal therapy compared to chemotherapy alone in terms of median progression-free survival (mPFS) and $\mathrm{OS}(\mathrm{mOS})$ from diagnosis of recurrence disease and of cFTM/BEV versus sFTM/BEV in terms of mPFS and mOS from the start of chemotherapy. Median PFS and OS were estimated with a $95 \%$ confidence interval. Survival curves of PFS and OS were generated using the Kaplan-Meier method. Differences in PFS and OS were evaluated using the log-rank test (Mantel-Cox) for statistical significance, which was defined at the $\mathrm{P}<0.05$ level (33).

Subgroup analyses according to treatment and $\mathrm{O}^{6}$-methylguanine-DNA methyltransferase (MGMT) and isocitrate dehydrogenase 1 (IDH-1) status were performed. Other subgroup analyses according to surgery, radiotherapy and other biological markers were not possible to perform due to the low number of patients.

Toxicity evaluation. All adverse events were graded according to NCI-CTCAE, version 4.03 (34). Toxicity assessment was performed at each cycle or, if clinically indicated, at weekly intervals. Evaluation of quality of life was not performed due to the lack of questionnaires in clinical practice.

\section{Results}

Patient characteristics. Twenty-six MGs patients treated at first relapse with multimodal therapy or chemotherapy were included in the analysis. Patients' characteristics are summarised in Table I. The two treatment groups are balanced for demographic and clinical characteristics. 
Table I. Patient characteristics and treatment at recurrence $(n=26)$.

A, Patient characteristics at recurrence

\begin{tabular}{|c|c|}
\hline Characteristics & $\mathrm{n}(\%)$ \\
\hline \multicolumn{2}{|l|}{ Sex } \\
\hline Male & $16(62)$ \\
\hline Female & $10(38)$ \\
\hline Median age, years (range) & $50(26-67)$ \\
\hline \multicolumn{2}{|l|}{ Karnofsky performance status } \\
\hline Median (range) & $80(60-100)$ \\
\hline $90-100$ & $9(35)$ \\
\hline $70-80$ & $16(61)$ \\
\hline 60 & $1(4)$ \\
\hline \multicolumn{2}{|l|}{ Laterality } \\
\hline Right & $11(42)$ \\
\hline Left & $15(58)$ \\
\hline \multicolumn{2}{|l|}{ Lobe } \\
\hline Fronto-temporal & $7(27)$ \\
\hline Parieto-temporal & $5(19)$ \\
\hline Monolobar & $13(50)$ \\
\hline Multilobar & $1(4)$ \\
\hline \multicolumn{2}{|l|}{ Histotype } \\
\hline Primary GBM & $20(77)$ \\
\hline Secondary GBM & $6(23)$ \\
\hline \multicolumn{2}{|c|}{ MGMT methylation status at diagnosis } \\
\hline Methylated & $16(62)$ \\
\hline Unmethylated & $10(38)$ \\
\hline \multicolumn{2}{|l|}{ IDH-1 status at diagnosis } \\
\hline Mutated & $5(19)$ \\
\hline Non mutated & $9(36)$ \\
\hline Unknown & $12(46)$ \\
\hline \multicolumn{2}{|l|}{ First-line therapy } \\
\hline Stupp protocol (RT/TMZ-TMZ) & $26(100)$ \\
\hline
\end{tabular}

$\mathrm{B}$, Treatment at recurrence

Treatment

$\mathrm{n}(\%)$

Type of treatment at recurrence

Multimodal therapy

Monotherapy

$11(42)$

$15(58)$

Surgery at recurrence

Yes

7 (27)

No

$19(73)$

Reirradiation at recurrence

Yes

No

Type of reirradiation

Photon-therapy

Proton-therapy

Chemotherapy at recurrence

$\mathrm{BEV}+\mathrm{FTM}$

$\mathrm{FTM} \rightarrow \mathrm{BEV}$
Table I. Continued.

$\mathrm{B}$, Treatment at recurrence

$\begin{array}{ll}\text { Treatment n }(\%) & \end{array}$

No. of median cycles

of chemotherapy received (range)

$$
\begin{gathered}
\mathrm{BEV}+\mathrm{FTM} \\
\mathrm{FTM} \rightarrow \mathrm{BEV} \\
\mathrm{FTM} \\
\mathrm{BEV}
\end{gathered}
$$

GBM, glioblastoma multiforme; MGMT, O6-methylguanine-DNA methyltransferase; IDH-1, isocitrate dehydrogenase 1; RT, radiotherapy; TMZ, temozolomide; BEV, bevacizumab; FTM, fotemustine.

Most patients were male (62\%), median age was 50 years (range, 26-67 years) and median KPS was 80 (range, 60-100). All patients had a histological diagnosis of MGs (77\% GBM and $23 \%$ grade-III gliomas). At first relapse all grade-III gliomas evolved into GBM (secondary GBM), a diagnosis that was made radiologically in 5 patients and histologically after resurgery in 1 patient.

The assessment of MGMT promoter status was conducted in all patients and resulted methylated in 16 patients $(62 \%)$ and unmethylated in 10 patients (38\%). The assessment of IDH status was conducted in 14 patients (54\%) and resulted mutated in 5 patients $(19 \%)$ and wild-type in 9 patients $(35 \%)$.

Fifteen patients $(58 \%)$ received chemotherapy alone and 11 patients $(42 \%)$ received multimodal therapy. Of these, 2 patients $(8 \%)$ underwent surgery followed by chemotherapy, 4 patients $(15 \%)$ received reirradiation followed by chemotherapy and 5 patients (19\%) underwent surgery followed by reirradiation and then chemotherapy. Twelve patients (46\%) were treated with $\mathrm{cFTM} / \mathrm{BEV}$ and 14 patients (54\%) with sFTM/BEV.

Treatment response evaluation. All patients included in the study were assessable for response analysis (Table II). Multimodal therapy showed 1 vs. 0 CR (9 vs. 0\%), 7 vs. 7 PR (64 vs. $47 \%$ ) and 1 vs. 3 SD (9 vs. 20\%) compared to chemotherapy alone. ORR and DCR of multimodal therapy were 73 and $82 \%$ compared to 47 and $67 \%$ with chemotherapy alone, respectively. Concomitant FTM/BEV resulted in 1 vs. $0 \mathrm{CR}$ ( 8 vs. $0 \%$ ), 9 vs. 5 PR (76 vs. $36 \%$ ), 1 vs. 3 SD ( 8 vs. $21 \%$ ) compared to sFTM/BEV. ORR and DCR of cFTM/BEV were of 84 and $92 \%$ respectively compared to 36 and $57 \%$ of sFTM/BEV.

General survival outcomes. All patients included in the study were assessable for survival analysis (Table II). Median PFS and OS from diagnosis of recurrence were 9 months (95\% CI 6.5-11.5) and 11 months (95\% CI 9.1-12.9) respectively, whereas mPFS and mOS from the start of chemotherapy were 7.1 months (95\% CI 5.6-8.6) and 9.5 months (95\% CI 5.1-13.9), respectively. 
Table II. Results for objective response and survival outcomes according to type of approach and treatment.

\begin{tabular}{|c|c|c|c|c|}
\hline Variables & $\begin{array}{c}\text { Multimodal } \\
\text { therapy }(n=11)\end{array}$ & $\begin{array}{l}\text { Monotherapy } \\
\qquad(\mathrm{n}=15)\end{array}$ & $\begin{array}{c}\text { Concomitant } \\
\text { FTM/BEV }(n=12)\end{array}$ & $\begin{array}{c}\text { Sequential } \\
\text { FTM/BEV }(n=14)\end{array}$ \\
\hline \multicolumn{5}{|c|}{ Objective response, $\mathrm{n}(\%)$} \\
\hline $\mathrm{CR}$ & $1(9 \%)$ & $0(0 \%)$ & $1(8 \%)$ & $0(0 \%)$ \\
\hline PR & $7(64 \%)$ & $7(47 \%)$ & $9(76 \%)$ & $5(36 \%)$ \\
\hline $\mathrm{SD}$ & $1(9 \%)$ & $3(20 \%)$ & $1(8 \%)$ & $3(21 \%)$ \\
\hline PD & $2(18 \%)$ & $5(33 \%)$ & $1(8 \%)$ & $6(43 \%)$ \\
\hline ORR & 73 & 47 & 84 & 36 \\
\hline $\mathrm{DC}$ & 82 & 67 & 92 & 57 \\
\hline \multicolumn{5}{|l|}{ Survival data } \\
\hline 6 months-PFS, $\%$ & 82 & 67 & 92 & 71 \\
\hline 12 months-PFS, \% & 27 & 20 & 25 & 21 \\
\hline Median PFS, months & 11 & 7 & 10 & 5 \\
\hline 6 months-OS, $\%$ & 91 & 73 & 83 & 50 \\
\hline 12 months-OS, $\%$ & 55 & 20 & 25 & 21 \\
\hline Median OS, months & 13 & 8 & 11 & 5.2 \\
\hline
\end{tabular}

$\mathrm{CR}$, complete response; $\mathrm{PR}$, partial response; $\mathrm{SD}$, stable disease; PD, progressive disease; ORR, overall response rate; DC, disease control; FTM, fotemustine; BEV, bevacizumab; PFS, progression-free survival; OS, overall survival.

Survival outcomes according to treatment. Multimodal therapy reported better survival outcomes in terms of mPFS and mOS compared to chemotherapy alone. Median PFS was 11 months (95\% CI 8-14) vs. 7 months $(95 \%$ CI 4.2-9.8) $(\mathrm{P}=0.08)$ and mOS was 13 months (95\% CI 11.2-14.8) vs. 8 months (95\% CI 5.5-10.5) ( $\mathrm{P}=0.04)$ (Fig. 1A and B). Concomitant FTM/BEV was associated with better survival outcomes in terms of mPFS of 10 months (95\% CI 8.6-11.4) versus 5 months (95\% CI 1-9) and mOS of 11 (95\% CI 10.3-11.7) vs. 5.2 months (95\% CI 1.7-8.7) compared to sFTM/BEV ( $\mathrm{P}=0.22$ and $\mathrm{P}=0.15$, respectively) (Fig.1C and D).

Activity according to MGMT status. Methylated patients $(n=16)$ experienced longer survival from the diagnosis of recurrence (both mPFS and mOS of 11 vs. 6 months; $\mathrm{P}=0.03$ and $\mathrm{P}=0.05$, respectively) and from the start of chemotherapy (mPFS: 8.2 vs. 3.8 months, $\mathrm{P}=0.11$; mOS: 10.6 vs. 5 months, $\mathrm{P}=0.08$ ), independently of the type of treatment. In methylated patients, multimodal treatment $(n=8)$ was associated with similar mPFS (both 11 months) and mOS (12 vs. 11 months) compared to chemotherapy alone $(n=8)$. Methylated patients experienced greater mPFS (10 vs. 7.1 months; $\mathrm{P}=0.33)$ and mOS (11 vs. 8 months; $\mathrm{P}=0.33)$ with cFTM/BEV $(\mathrm{n}=6)$ compared to sFTM/BEV ( $\mathrm{n}=10)$ (Fig. $2 \mathrm{~A}$ and $\mathrm{B})$.

The greatest benefit was observed in unmethylated patients who experienced statistically significant longer survival with multimodal therapy and $\mathrm{cFTM} / \mathrm{BEV}$. Unmethylated patients experienced higher mPFS (10 vs. 5 months; $\mathrm{P}=0.02$ ) and $\mathrm{mOS}$ (11 vs. 6 months; $\mathrm{P}=0.02)$ with multimodal therapy $(\mathrm{n}=3)$ compared to chemotherapy alone $(n=7)$ and greater mPFS (5 vs. 2 months; $\mathrm{P}=0.01$ ) and $\operatorname{mOS}(6$ vs. 3.2 months; $\mathrm{P}=0.01$ ) with cFTM/BEV $(n=6)$ compared to sFTM/BEV $(n=4)$ (Fig. 2C and D).
Toxicity evaluation. All patients were evaluated for safety. Concomitant FTM/BEV was well-tolerated with grade 1-2 myelotoxicities in 62 vs. $70 \%$ of patients, grade 3 myelotoxicity in 8 vs. $15 \%$ of patients and grade 1-2 hypertransaminasemia in 23 vs. $38 \%$ of patients compared to sFTM/BEV. Grade 1-2 fatigue was present in $30 \%$ of patients in both treatments. Grade 1-2 hypertension and proteinuria developed in 10 and $15 \%$ of patients in cFTM/BEV vs. $20 \%$ and $40 \%$ of patients in sFTM/BEV. No grade 4 adverse events were observed. None of the patients discontinued for toxicity.

\section{Discussion}

For recurrent MGs, different treatment strategies are available, such as resurgery, reirradiation and systemic chemotherapy, as well as their combinations, depending on clinical status, tumour location and extension and time interval since last treatment. Nonetheless, the optimal management of recurrent MGs has not yet been established, which represents a marked clinical challenge.

Local recurrence within $2 \mathrm{~cm}$ of the resection bed of the primary tumour is the most common pattern of failure. Therefore, local strategies such as surgical resection and/or radiotherapy in combination with systemic chemotherapy, in a multidisciplinary approach, may offer an advantage in local control and may improve survival outcomes.

Recent literature reviews and several retrospective studies suggest a survival benefit with reoperation at the time of recurrence. Favourable PS and extent of resection (gross total resection vs. partial surgery) are the main predictors of survival (5-10) and the addition of adjuvant treatments (chemotherapy and radiosurgery) prolongs survival $(5,35,36)$. 

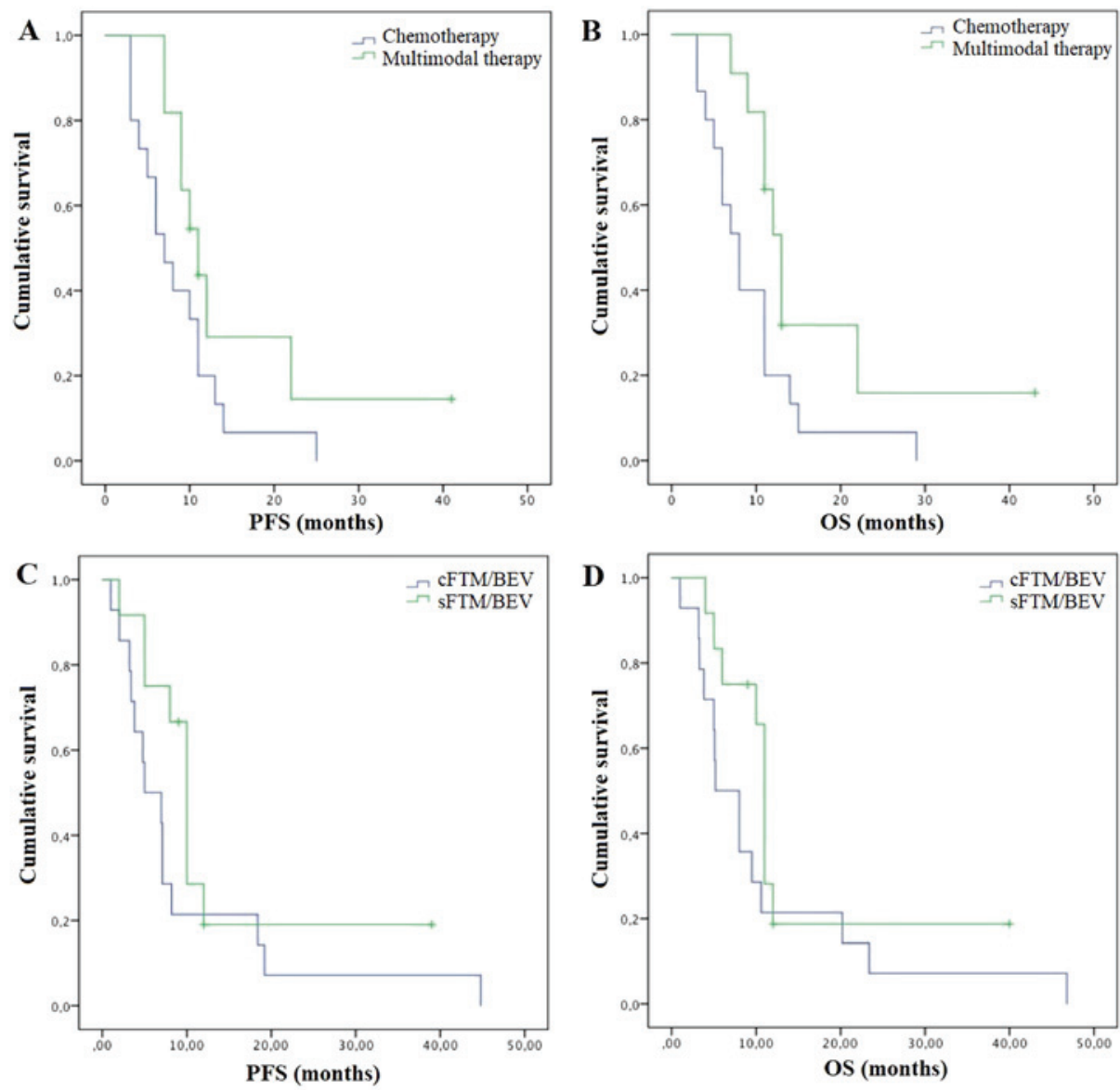

Figure 1. Survival outcomes according to treatment. Kaplan-Meier curves for (A) PFS and (B) OS in MGs patients treated with multimodal therapy compared to chemotherapy alone and for (C) PFS and (D) OS in MGs patients treated with concomitant FTM/BEV compared to sequential FTM/BEV. PFS, progression-free survival; OS, overall survival; MGs, malignant gliomas; FTM, fotemustine; BEV, bevacizumab.
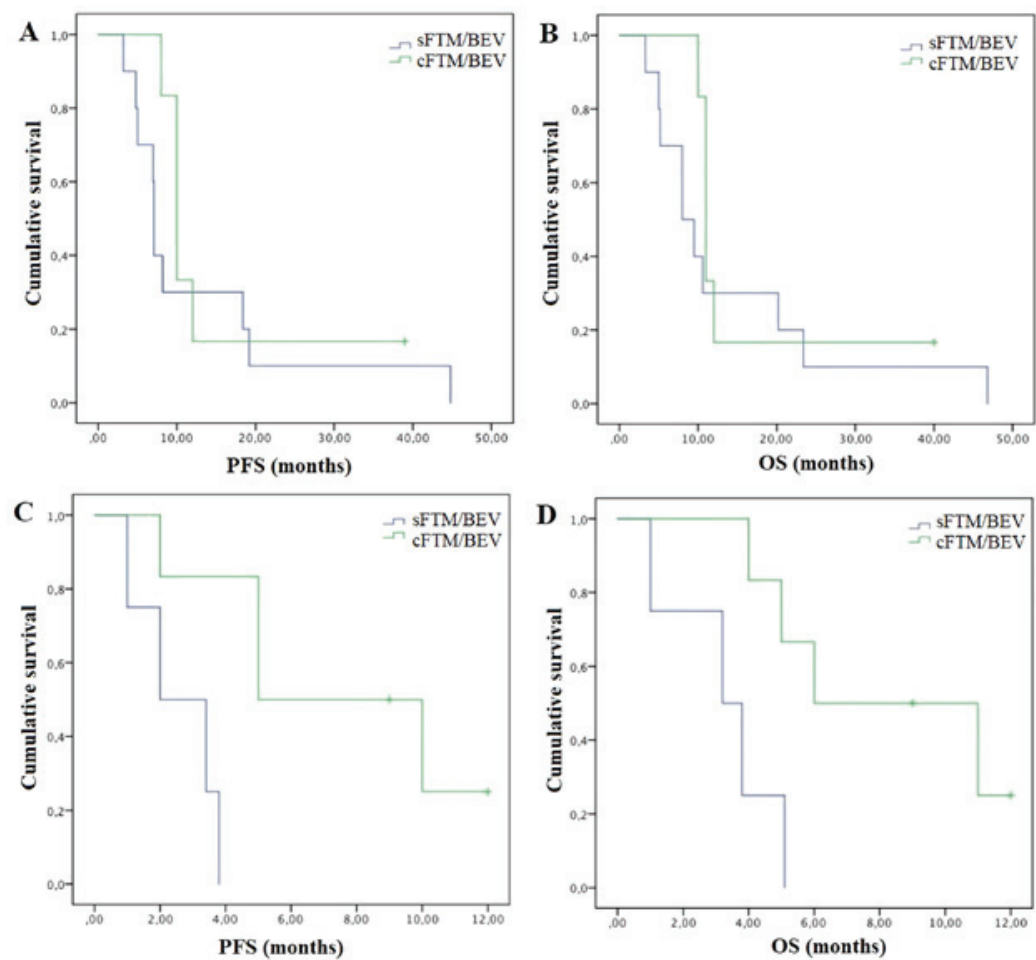

Figure 2. Survival outcomes according to MGMT-methylation. Kaplan-Meier curves for (A) PFS and (B) OS in methylated patients and for (C) PFS and (D) OS in non-methylated patients treated with concomitant FTM/BEV compared to sequential FTM/BEV. MGMT, $\mathrm{O}^{6}$-methylguanine-DNA methyltransferase; PFS, progression-free survival; OS, overall survival; MGs, malignant gliomas; FTM, fotemustine; BEV, bevacizumab. 

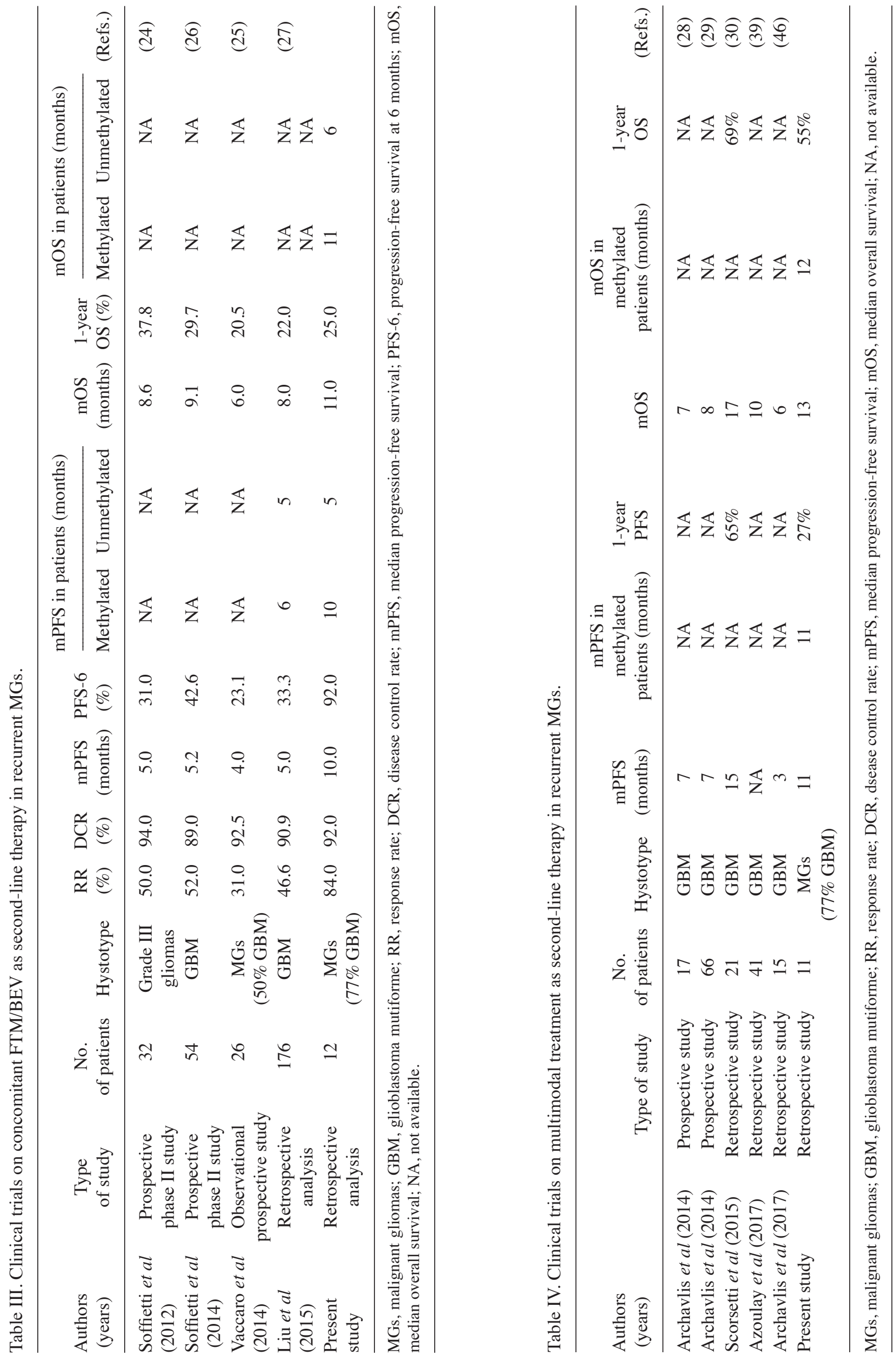
Several trials suggest an improvement in survival and functional status with local reirradiation in younger patients with good PS, tumour size $<4 \mathrm{~cm}$ and progression more than 6 months from first irradiation $(14,37,38)$. Retrospective and prospective trials have investigated the benefits of reirradiation as adjuvant therapy after resurgery (36) or as part of a combined approach with chemotherapy (39). There is no consensus on one particular radiation regimen, but higher doses per fraction with modern precision radiotherapy (PBT, fractionated stereotactic radiotherapy or stereotactic radiosurgery), are associated with smaller recurrences and clinical efficacy with low toxicity rates. Systemic agents used as radiosensitizers in combination with radiotherapy are cytotoxic and targeted systemic agents, such as TMZ and BEV (38).

Systemic therapy, consisting of chemotherapeutic and anti-angiogenetic drugs, is the main treatment employed and investigated for recurrent gliomas as single agents or as combination regimens $(21,30)$, but the optimal combination and sequencing have not yet been established. The most used drugs are rechallenge TMZ, nitrosoureas and BEV (40).

According to several systematic reviews and metaanalyses, BEV as a single agent and in combination with chemotherapy, both as first or second-line treatments, has been shown to be effective in terms of ORR, PFS and reducing symptoms, but not in terms of OS $(5,41)$.

Interesting results were shown with the combination of BEV and nitrosoureas $(41,42)$, such as lomustine and FTM. The BELOB phase II trial (42), the subsequent phase III trial EORTC-26101 (43) and two recent trials $(44,45)$ showed that the combination BEV/lomustine at first recurrence was superior to $\mathrm{BEV}$ or lomustine monotherapy (41). Other interesting results were obtained by retrospective and prospective trials on the combination of BEV and FTM (24-27) (Table III). Soffietti et al. showed the efficacy of the association of BEV/FTM at first recurrence in recurrent grade-III gliomas (24) and GBM patients, in terms of survival outcome and response rate (26). Similar results were reported by a retrospective analysis conducted by Liu et al (27) and an observational prospective study by Vaccaro et al (25).

Several studies showed that the efficacy of systemic chemotherapy in terms of disease control and survival is improved by the combination with local treatments such as surgery and/or irradiation (28-30,39,46) (Table IV). In 2015 Scorsetti et al (30) evaluated 43 GBM patients treated by chemotherapy plus local treatment or chemotherapy alone, showing that the combined treatment achieved better survival results in terms of PFS (15 vs. 5 months) and OS (17 vs. 6 months).

Azoulay et al (39) conducted a retrospective study to assess the benefits of resurgery followed by chemotherapy and/or reirradiation compared to resurgery alone and chemotherapy and/ or reirradiation. Median survival was superior in the multimodal treatment compared to the other treatment approaches (10 vs. 6.8 vs. 6.6 months).

Archavlis et al. showed in three clinical studies $(28,29,46)$ that a combined therapy of resurgery, brachytherapy and chemotherapy achieved better survival outcomes compared to a historical control group of patients treated with TMZ.

We report our experience with the multimodal management of recurrent MGs, as the combination of resurgery and/or radiotherapy and chemotherapy, compared to chemotherapy alone. In regards to chemotherapy, we studied the efficacy of cFTM/BEV compared to sFTM/BEV, an idea born from the study of Piccioni et al (47), which demonstrated equal efficacy of BEV monotherapy on first, second or third recurrence in recurrent GBM.

We observed that multimodal therapy was associated with $25 \%$ higher response rates, $15 \%$ higher DCR and a survival improvement of 4 months in PFS and 5 months in OS compared to chemotherapy alone. Our results are in line with those reported by other retrospective and prospective trials on multimodal treatment (Table IV), showing the possibility of combining systemic chemotherapy with local treatment to improve local control of the disease and survival outcomes.

According to the type of chemotherapy, we observed $\sim 50 \%$ higher response rates, 35\% higher DCR and better survival outcomes with cFTM/BEV compared to sFTM/BEV. Our results seem to be stronger than those reported by other trials regarding cFTM/BEV (Table III), which was probably due to the addition of reirradiation, alone or after surgery.

Only recently, the DIRECTOR and the BELOB trials demonstrated the prognostic value of the MGMT methylation also in recurrent GBM $(42,48,49)$. The AVAREG trial $(50)$ demonstrated also that MGMT methylation status was predictive of efficacy of FTM in the recurrence setting. We observed an association between MGMT methylation and longer survival independent of the type of treatment. Methylated patients appear not to benefit from a multimodal approach, but a survival benefit was observed with the combination therapy compared to FTM alone, whereas unmethylated patients appear to benefit from both multimodal therapy and concomitant systemic therapy better than methylated patients. Subgroup analyses on MGMT methylation in this setting were not reported in other similar clinical trials (Table III-IV). Similar to the other trials on cFTM/BEV, the combination therapy was well-tolerated, with most frequent grade 3-4 toxicities related to chemotherapy.

The main limitations of this study are the low number of patients with small subgroups resulting in a lack of statistically significant results, the heterogeneity and non-standardisation in the therapeutic approach used, and the retrospective and non-randomised nature, resulting in possible selection biases for each treatment modality.

Despite these limitations our encouraging survival and local control results underlined that the management of recurrent MGs patients, especially those with a poorer survival such as unmethylated patients, should involve a multidisciplinary approach, associating local treatments (surgery and/or radiotherapy) to chemotherapy, or a combination of chemotherapies, whenever possible. Moreover, in this multimodal view of the treatment of MGs patients, molecular characteristics play a relevant role in the decision making to determine the best choice of treatment and the highest survival benefit possible.

Considering that no optimal treatment combinations and sequencing have been established, our results could be a starting point for further larger prospective studies.

In conclusion, our experience showed that in MG patients at first recurrence, multimodal treatment (chemotherapy plus surgery and/or radiotherapy) achieves better survival and response results compared to chemotherapy alone. Moreover, concomitant BEV/FTM provides higher survival benefit and 
response rates, without adding higher toxicity, compared to the sequential approach. Better survival outcomes were observed in MGMT methylated patients but MGMT unmethylated patients have shown a greater survival benefit with both multimodal therapy and cFTM/BEV.

\section{Acknowledgements}

Not applicable.

\section{Funding}

No funding was received.

\section{Availability of data and materials}

The datasets used and/or analysed during the current study are available from the corresponding author on reasonable request.

\section{Authors' contributions}

AP and SER were the major contributors in writing the manuscript, analysing and interpreting the patient data. MG, JRGB, $\mathrm{SP}$ and $\mathrm{CF}$ were involved in acquisition, analysis and interpretation of patient data. MS, ST, VB were involved in writing the manuscript and revising it critically for important intellectual content. All authors read and approved the final manuscript.

\section{Ethics approval and consent to participate}

All procedures performed in studies involving human participants were in accordance with the ethical standards of the institutional and/or national research committee and with the 1964 Helsinki declaration and its later amendments or comparable ethical standards. Informed consent was obtained from all individual participants included in the study.

\section{Patient consent for publication}

Not applicable.

\section{Competing interests}

The authors declare that they have no competing interests.

\section{References}

1. Ostrom QT, Gittleman H, Farah P, Ondracek A, Chen Y, Wolinsky Y, Stroup NE, Kruchko C and Barnholtz-Sloan JS: CBTRUS statistical report: primary brain and central nervous system tumors diagnosed in the United States in 2006-2010. Neuro Oncol 15 (Sup 6): ii1-ii56, 2013.

2. Stupp R, Mason WP, van den Bent MJ, Weller M, Fisher B, Taphoorn MJ, Belanger K, Brandes AA, Marosi C, Bogdahn U, et al; European Organisation for Research and Treatment of Cancer Brain Tumor and Radiotherapy Groups; National Cancer Institute of Canada Clinical Trials Group: Radiotherapy plus concomitant and adjuvant temozolomide for glioblastoma. $\mathrm{N}$ Engl J Med 352: 987-996, 2005.

3. Delgado-López PD and Corrales-García EM: Survival in glioblastoma: A review on the impact of treatment modalities. Clin Transl Oncol 18: 1062-1071, 2016.
4. Weller M, van den Bent M, Hopkins K, Tonn JC, Stupp R, Falini A, Cohen-Jonathan-Moyal E, Frappaz D, Henriksson R, Balana C, et al; European Association for Neuro-Oncology (EANO) Task Force on Malignant Glioma: EANO guideline for the diagnosis and treatment of anaplastic gliomas and glioblastoma. Lancet Oncol 15: e395-e403, 2014.

5. Montemurro N, Perrini P, Blanco MO and Vannozzi R: Second surgery for recurrent glioblastoma: A concise overview of the current literature. Clin Neurol Neurosurg 142: 60-64, 2016.

6. Tosoni A, Franceschi E, Poggi R and Brandes AA: Relapsed Glioblastoma: Treatment Strategies for Initial and Subsequent Recurrences. Curr Treat Options Oncol 17: 49, 2016.

7. Hervey-Jumper SL and Berger MS: Reoperation for recurrent high-grade glioma: A current perspective of the literature. Neurosurgery 75: 491-499, discussion 498-499, 2014.

8. Davis ME: Glioblastoma: Overview of Disease and Treatment. Clin J Oncol Nurs 20: S2-S8, 2016.

9. Brandes AA, Bartolotti M, Tosoni A, Poggi R, Bartolini S, Paccapelo A, Bacci A, Ghimenton C, Pession A, Bortolotti C, et al: Patient outcomes following second surgery for recurrent glioblastoma. Future Oncol 12: 1039-1044, 2016.

10. Suchorska B, Weller M, Tabatabai G, Senft C, Hau P, Sabel MC, Herrlinger U, Ketter R, Schlegel U, Marosi C, et al: Complete resection of contrast-enhancing tumor volume is associated with improved survival in recurrent glioblastoma-results from the DIRECTOR trial. Neuro-oncol 18: 549-556, 2016.

11. Gorlia T, Stupp R, Brandes AA, Rampling RR, Fumoleau P, Dittrich C, Campone MM, Twelves CC, Raymond E, Hegi ME, et al: New prognostic factors and calculators for outcome prediction in patients with recurrent glioblastoma: A pooled analysis of EORTC Brain Tumour Group phase I and II clinical trials. Eur J Cancer 48: 1176-1184, 2012.

12. Nava F, Tramacere I, Fittipaldo A, Bruzzone MG, Dimeco F, Fariselli L, Finocchiaro G, Pollo B, Salmaggi A, Silvani A, et al: Survival effect of first- and second-line treatments for patients with primary glioblastoma: A cohort study from a prospective registry, 1997-2010. Neuro-oncol 16: 719-727, 2014.

13. Taunk NK, Moraes FY, Escorcia FE, Mendez LC, Beal K and Marta GN: External beam re-irradiation, combination chemoradiotherapy, and particle therapy for the treatment of recurrent glioblastoma. Expert Rev Anticancer Ther 16: 347-358, 2016.

14. Sulman EP, Ismaila N, Armstrong TS, Tsien C, Batchelor TT, Cloughesy T, Galanis E, Gilbert M, Gondi V, Lovely M, et al: Radiation Therapy for Glioblastoma: American Society of Clinical Oncology Clinical Practice Guideline Endorsement of the American Society for Radiation Oncology Guideline. J Clin Oncol 35: 361-369, 2017.

15. Mizumoto M, Yamamoto T, Ishikawa E, Matsuda M, Takano S, Ishikawa H, Okumura T, Sakurai H, Matsumura A and Tsuboi K: Proton beam therapy with concurrent chemotherapy for glioblastoma multiforme: Comparison of nimustine hydrochloride and temozolomide. J Neurooncol 130: 165-170, 2016.

16. Minniti G, Armosini V, Salvati M, Lanzetta G, Caporello P, Mei M, Osti MF and Maurizi RE: Fractionated stereotactic reirradiation and concurrent temozolomide in patients with recurrent glioblastoma. J Neurooncol 103: 683-691, 2011.

17. Adeberg S, Harrabi SB, Bougatf N, Bernhardt D, Rieber J, Koerber SA, Syed M, Sprave T, Mohr A, Abdollahi A, et al: Intensity-modulated proton therapy, volumetric-modulated arc therapy, and 3D conformal radiotherapy in anaplastic astrocytoma and glioblastoma: A dosimetric comparison. Strahlenther Onkol 192: 770-779, 2016.

18. Matsuda M, Yamamoto T, Ishikawa E, Nakai K, Zaboronok A, Takano S and Matsumura A: Prognostic factors in glioblastoma multiforme patients receiving high-dose particle radiotherapy or conventional radiotherapy. Br J Radiol 84: S54-S60, 2011.

19. Mizumoto M, Okumura T, Ishikawa E, Yamamoto T, Takano S, Matsumura A, Oshiro Y, Ishikawa $\mathrm{H}$, Sakurai $\mathrm{H}$ and Tsuboi $\mathrm{K}$ : Reirradiation for recurrent malignant brain tumor with radiotherapy or proton beam therapy. Technical considerations based on experience at a single institution. Strahlenther Onkol 189: 656-663, 2013.

20. Galle JO, McDonald MW, Simoneaux V and Buchsbaum JC: Reirradiation with proton therapy for recurrent gliomas. Int $\mathrm{J}$ Part Ther 2: 11-18, 2015.

21. Seystahl K, Wick W and Weller M: Therapeutic options in recurrent glioblastoma-An update. Crit Rev Oncol Hematol 99: 389-408, 2016. 
22. Wang Y, Xing D, Zhao M, Wang J and Yang Y: The role of a single angiogenesis inhibitor in the treatment of recurrent glioblastoma multiforme: A meta-analysis and systematic review. PLoS One 11: e0152170, 2016.

23. Addeo R, Caraglia M, De Santi MS, Montella L, Abbruzzese A, Parlato C, Vincenzi B, Carraturo M, Faiola V, Genovese M, et al: A new schedule of fotemustine in temozolomide-pretreated patients with relapsing glioblastoma. J Neurooncol 102: 417-424, 2011

24. Soffietti R, Trevisan E, Bosa C, Bertero L and Ruda R: Phase II trial of bevacizumab and fotemustine in recurrent grade III gliomas. J Clin Oncol 30: Abstract 2075, 2012.

25. Vaccaro V, Fabi A, Vidiri A, Giannarelli D, Metro G, Telera S, Vari S, Piludu F, Carosi MA, Villani V, et al: Activity and safety of bevacizumab plus fotemustine for recurrent malignant gliomas. BioMed Res Int 2014: 351252, 2014.

26. Soffietti R, Trevisan E, Bertero L, Cassoni P, Morra I, Fabrini MG, Pasqualetti F, Lolli I, Castiglione A, Ciccone G, et al: Bevacizumab and fotemustine for recurrent glioblastoma: A phase II study of AINO (Italian Association of NeuroOncology). J Neurooncol 116: 533-541, 2014

27. Liu Z, Zhang G, Zhu L, Wang J, Liu D, Lian L, Liu J, Lai T and Zhuang X: Retrospective analysis of bevacizumab in combination with fotemustine in chinese patients with recurrent glioblastoma multiforme. Biomed Res Int 2015: 723612, 2015.

28. Archavlis E, Tselis N, Birn G, Ulrich P and Zamboglou N: Salvage therapy for recurrent glioblastoma multiforme: A multimodal approach combining fluorescence-guided resurgery, interstitial irradiation, and chemotherapy. Neurol Res 36: 1047-1055, 2014.

29. Archavlis E, Tselis N, Birn G, Ulrich P and Zamboglou N: Combined salvage therapies for recurrent glioblastoma multiforme: Evaluation of an interdisciplinary treatment algorithm. J Neurooncol 119: 387-395, 2014.

30. Scorsetti M, Navarria P, Pessina F, Ascolese AM, D'Agostino G, Tomatis S, De Rose F, Villa E, Maggi G, Simonelli M, et al: Multimodality therapy approaches, local and systemic treatment, compared with chemotherapy alone in recurrent glioblastoma. BMC Cancer 15: 486, 2015

31. Louis DN, Perry A, Reifenberger G, von Deimling A, FigarellaBranger D, Cavenee WK, Ohgaki H, Wiestler OD, Kleihues P and Ellison DW: The 2016 World Health Organization Classification of Tumors of the Central Nervous System: A summary. Acta Neuropathol 131: 803-820, 2016

32. Wen PY, Macdonald DR, Reardon DA, Cloughesy TF, Sorensen AG, Galanis E, Degroot J, Wick W, Gilbert MR, Lassman AB, et al: Updated response assessment criteria for high-grade gliomas: Response assessment in neuro-oncology working group. J Clin Oncol 28: 1963-1972, 2010

33. Kaplan E and Meier P: Nonparametric estimation from incomplete observations. J Am Stat Assoc 53: 457-481, 1958.

34. Common Terminology Criteria for Adverse Events v4.03 (CTCAE): http://ctep.cancer.gov. Accessed July 22, 2017

35. Mandl ES, Dirven CM, Buis DR, Postma TJ and Vandertop WP: Repeated surgery for glioblastoma multiforme: Only in combination with other salvage therapy. Surg Neurol 69: 506-509, discussion 509, 2008

36. Straube C, Elpula G, Gempt J, Gerhardt J, Bette S, Zimmer C, Schmidt-Graf F, Meyer B and Combs SE: Re-irradiation after gross total resection of recurrent glioblastoma : Spatial pattern of recurrence and a review of the literature as a basis for target volume definition. Strahlenther Onkol 193: 897-909, 2017.

37. Cabrera AR, Kirkpatrick JP, Fiveash JB, Shih HA, Koay EJ, Lutz S, Petit J, Chao ST, Brown PD, Vogelbaum M, et al: Radiation therapy for glioblastoma: Executive summary of an American Society for Radiation Oncology Evidence-Based Clinical Practice Guideline. Pract Radiat Oncol 6: 217-225, 2016.

38. Howard SP, Krauze A, Chan MD, Tsien C and Tomé WA: The evolving role for re-irradiation in the management of recurrent grade 4 glioma. J Neurooncol 134: 523-530, 2017.
39. Azoulay M, Santos F, Shenouda G, Petrecca K, Oweida A, Guiot MC, Owen S, Panet-Raymond V, Souhami L and Abdulkarim BS: Benefit of re-operation and salvage therapies for recurrent glioblastoma multiforme: Results from a single institution. J Neurooncol 132: 419-426, 2017.

40. National Comprehensive Cancer Network: NCCN Clinical Practice Guidelines in Oncology (NCCN Guidelines ${ }^{\circledR}$ ): Central Nervous System Cancers. NCCN Evidence Blocks. Version 1.2017. https://www.nccn.org/professionals/physician_ gls/pdf/cns_blocks.pdf. Accessed December 5, 2017.

41. Lombardi G, Pambuku A, Bellu L, Farina M, Della Puppa A, Denaro L and Zagonel V: Effectiveness of antiangiogenic drugs in glioblastoma patients: A systematic review and meta-analysis of randomized clinical trials. Crit Rev Oncol Hematol 111: 94-102, 2017

42. Taal W, Oosterkamp HM, Walenkamp AM, Dubbink HJ, Beerepoot LV, Hanse MC, Buter J, Honkoop AH, Boerman D, de Vos FY, et al: Single-agent bevacizumab or lomustine versus a combination of bevacizumab plus lomustine in patients with recurrent glioblastoma (BELOB trial): A randomised controlled phase 2 trial. Lancet Oncol 15: 943-953, 2014.

43. Wick W, Brandes AA, Gorlia T, Bendszus M, Sahm F, Taal W, Taphoorn M, Domont J, Idbaih A, Campone M, et al: Phase III trial exploring the combination of bevacizumab and lomustine in patients with first recurrence of a glioblastoma: The EORTC 26101 trial. Neuro-oncol 17 (Suppl 5): LB05, 2015.

44. Weathers SP, Han X, Liu DD, Conrad CA, Gilbert MR, Loghin ME, O'Brien BJ, Penas-Prado M, Puduvalli VK, Tremont-Lukats I, et al: A randomized phase II trial of standard dose bevacizumab versus low dose bevacizumab plus lomustine (CCNU) in adults with recurrent glioblastoma. J Neurooncol 129: 487-494, 2016.

45. Heiland DH, Masalha W, Franco P, Machein MR and Weyerbrock A: Progression-free and overall survival in patients with recurrent Glioblastoma multiforme treated with last-line bevacizumab versus bevacizumab/lomustine. J Neurooncol 126: 567-575, 2016.

46. Archavlis E: Combined Salvage Therapies for Recurrent Glioblastoma Multiforme: Treatment Options in Multifocal and Multicentric Patterns of Recurrence. J Cancer Prev Curr Res 7: 00222, 2017.

47. Piccioni DE, Selfridge J, Mody RR, Chowdhury R, Li S, Lalezari S, Wawrzynski J, Quan J, Zurayk M, Chou AP, et al: Deferred use of bevacizumab for recurrent glioblastoma is not associated with diminished efficacy. Neuro-oncol 16: 815-822, 2014.

48. Weller M, Tabatabai G, Kästner B, Felsberg J, Steinbach JP, Wick A, Schnell O, Hau P, Herrlinger U, Sabel MC, et al; DIRECTOR Study Group: MGMT promoter methylation is a strong prognostic biomarker for benefit from dose-intensified temozolomide rechallenge in progressive glioblastoma: The DIRECTOR Trial. Clin Cancer Res 21: 2057-2064, 2015.

49. Szopa W, Burley TA, Kramer-Marek G and Kaspera W: Diagnostic and Therapeutic Biomarkers in Glioblastoma: Current Status and Future Perspectives. BioMed Res Int 2017: 8013575, 2017.

50. Brandes AA, Finocchiaro G, Zagonel V, Reni M, Caserta C, Fabi A, Clavarezza M, Maiello E, Eoli M, Lombardi G, et al: AVAREG: A phase II, randomized, noncomparative study of fotemustine or bevacizumab for patients with recurrent glioblastoma. Neuro-oncol 18: 1304-1312, 2016.

This work is licensed under a Creative Commons Attribution-NonCommercial-NoDerivatives 4.0 International (CC BY-NC-ND 4.0) License. 